\title{
Animal Welfare in the Uruguayan Veterinary Profession
}

\section{Field}

\author{
Malvina María Prieto Laport ${ }^{1,2}$, Pablo Ernesto Bobadilla Robledo ${ }^{1,2}$ and Stella Maris Huertas Cánen ${ }^{1,2}$ \\ 1. Facultad de Veterinaria, Universidad de la República, Montevideo, Lasplaces 1550, CP 11600, Uruguay \\ 2. Centro Colaborador OIE en Bienestar Animal y Sistemas de Producción Pecuarios, Chile-Uruguay-México
}

\begin{abstract}
Training and dissemination of good handling practices are key points in reducing animal abuse. Three aspects are considered in the development of education in animal welfare (AW): (1) introduction of AW in curricular education, nevertheless it has not been applied in the Facultad de Veterinaria, Universidad de la República de Uruguay, up to now; (2) promotion of permanent education for graduates; (3) development of plans for dissemination, follow up, assessment and continuous improvement on AW affairs. Aiming to obtain information about the knowledge of veterinarians on AW during the practice of their professional duties, in September 2016, an on-line questionnaire was sent to the veterinarians who were in the Educación Continua database. The questionnaire had four sections: a general gathering information and three additional sections, one for each veterinarian professional field (large animal's health and production, small animal's health and food industry). From 1,804 questionnaires sent, 388 answers were obtained. There were $69 \%$ attended at least one AW course, and $89.9 \%$ were willing to attend another AW course. And $63.5 \%$ considered the information received to be very useful for their daily activities, 95\% considered that AW should be included in the veterinary studies curriculum, while 53.3\% declared not having knowledge about the regional animal welfare strategy (RAWS). The majority of the veterinarians considered the acquired knowledge very useful and are eager to obtain more information. It is necessary to keep working to include AW in the veterinary studies curriculum as well as continuing with the diffusion of good practices and AW principles, based on the national and international norms.
\end{abstract}

Key words: Education, veterinary curricula, animal welfare, veterinary profession, good handling practices, on-line survey.

\section{Introduction}

The veterinary profession plays a key role in guaranteeing animal health and welfare, for this, veterinary training needs to include a bigger variety of animal welfare (AW) related courses in the curricula $[1,2]$.

Since 2012, the World Organization for Animal Health (OIE) defined the minimum expected capabilities for the veterinary profession, where aspects related to new importance areas for AW are included [3, 4]. The veterinary community as well as each veterinarian should have the mission to guarantee animal health and welfare. [1, 5] For this, it is imperative for veterinarians not only to fulfill the traditional functions, but also to inform, educate and

Corresponding author: Malvina María Prieto Laport, Doctor, research field: veterinary science. make the different actors involved, such as the general public, farm owners and stockmen, aware of the issues related with responsible ownership and good handling practices [5-7]. Due to the challenges faced today to implement AW standards, it is important to use new effective strategies of education. In theory, education is one of the most relevant activities to promote AW [8].

According to some of the discussion results obtained in the meeting "Professional Profile of the Veterinary Medic in Latin America: Vision towards 2030" by the Animal Welfare Board, the veterinary medic of 2030 should contemplate certain globalizing knowledge on AW, that allows to articulate its technical education with ethical, moral, philosophical and socio-cultural aspects. To achieve this, education institutions should not only fulfill the pre-established curricular programs, but also cover other areas which 
are needed, such as mandatory AW courses [9]. Training and diffusion of good handling practices of animals constitute key elements to achieve substantial improvements and to reduce animal abuse, based on the policy of AW for the Americas [7]. In 2012, OIE proposes the regional animal welfare strategy (RAWS) for the Americas that includes the different aspects related to politics, science, ethics, economics and food safety of the countries involved. RAWS seeks to involve all the responsible actors to attain the maximum consensus, thus achieving a common vision and mission [10].

Three aspects are considered in the development of education in AW: (1) the introduction to AW in the curricular education; (2) the promotion of continuous education activities for graduates; (3) the development of dissemination, follow up, evaluation and continuous improvement plans in AW [11].

According to the latest national veterinary census, Uruguay has 3,304 active veterinarians, working in different professional fields: veterinary medicine (44\%), animal production (22\%), animal health (14\%), food technology (10\%) and public health (3\%) [12].

A partial survey of 250 veterinary graduates from the Facultad de Veterinaria, Universidad de la República (Fvet-UdelaR) database, showed that $47 \%$ attended to three or more AW courses and 65\% reported that AW concepts were very applicable to their job [13]. Nevertheless, AW is not yet included in the curricula of Fvet-UdelaR, the only institution were that veterinary studies can be undertaken in Uruguay.

The aim of the present study was to obtain more information on the veterinary graduates' knowledge about AW and its application in the practice of their professional duties.

\section{Materials and Methods}

During September 2016, a survey was conducted, using an on-line form, sent by email to 1,804 veterinary graduates who are included in the Programa de Educación Continua of the Fvet-UdelaR database.
The survey contained four sections, a general one gathering information and three additional sections, one for each veterinarian professional field (large animal's health and production, small animal's health and food industry), as shown in Table 1.

The on-line tool compiled in real time the answers submitted, and then organized the data obtained to allow a descriptive statistics analysis.

\section{Results and Discussion}

From the 1,804 on-line forms sent, 388 responses were obtained (21.5\%), accounting for $11.7 \%$ of the total veterinarians in the country [12].

The survey results showed that $69 \%$ attended at least one AW course and $63.5 \%$ considered the information received very useful for their daily activities. The similar results were reported by Huertas et al. [13]. The attendance to AW courses increased in comparison to a 2012 survey (Fig. 1). In the survey, 95\% of the respondents considered that AW should be included in the veterinary curricula and $89.9 \%$ were willing to attend another AW course if offered.

Half of the veterinarians surveyed (53.3\%) declared not having any knowledge about the RAWS. Even though this tool was initially presented at an academic level in 2014, its promotion has not had sufficient reach in the country [14].

Among veterinarians, $68.5 \%$ worked in animal production and/or large animal medicine and $14.6 \%$ in the food industry. These results match with the beef cattle oriented livestock production of Uruguay [7, 15]. However, 24\% of veterinarians worked with small animals (pets).

In the survey, $44 \%$ of veterinarians answered that $25 \%$ of the farms they visit had knowledge of and properly implemented good animal handling practices; $54.4 \%$ of responses considered the farm owner as the main information source of AW practices for farm staff, along with the veterinarian (50.3\%).

Table 2 shows the tools or methods used to herd the animals on the farms visited by the veterinarians. The 
Table 1 Questionnaire about “AW in Uruguay” sent by email to 1,804 veterinarians resident in Uruguay during the month of September 2016.

Dear colleague please answer the following questions by marking the slot that corresponds with your answer.

1. Mark your year of entry to the university
(a) Before 1980
(b) Between 1980 and 1990
(c) Between 1991 and 2001
(d) Between 2002 and 2012

2. Have you attended AW courses?
(a) Never
(b) Once
(c) Twice
(d) More than twice

3. The information received in the AW courses was useful for your daily work with animals?
(a) A lot
(b) Little
(c) Not at all

4. Are you willing to attend an AW related activity again?
(a) Yes

(b) No

5. Despite AW (as a subject) is not part of the mandatory study program, do you consider it necessary for the education of the current veterinarians?
(a) Yes
(b) No

6. Regarding the OIE regional strategy for AW, what's your level of knowledge of the RAWS?
(a) Heard about the strategy
(b) Have read the strategy and understand its reach
(c) Have read the strategy and do not understand its reach
(d) Do not know about the strategy

7. What's your work area?
(a) Animal production or large animals health (bovine, ovine and/or equine)
(b) Small animals (pets)
(c) Food industry (meat, dairy or other)
(d) Other

7a Animal production or large animals medicine (bovine, ovine and/or equine)
(1) When you work with animals, you use (mark all the options you use):
(a) Electric prod
(b) Sticks
(c) Shouts
(d) Flags
(e) Dogs
(f) None of the previous
(g) Other
(2) On the farms you visit, farm staff when working with animals use:
(a) Electric prod
(b) Sticks
(c) Shouts
(d) Flags
(e) Dogs
(f) None of the previous
(g) Other 
(Table 1 continued)

Dear colleague please answer the following questions by marking the slot that corresponds with your answer.

(3) On how many of the farms you visit, you consider them having knowledge about good animal handling practices/AW?

(a) None of them

(b) Less than $25 \%$

(c) Between $25 \%$ and $50 \%$

(d) Between $50 \%$ and $75 \%$

(e) More than $75 \%$

(f) In all of them

(4) Which do you consider to be the main source for information about AW for farm staff?

(a) Tradition

(b) Trainings

(c) Conversation with the farm technicians (including the veterinarian)

(d) Indications made by the farm owner

(e) None of the previous

7b Small animals (pets)

(1) You mainly work in neighborhoods of which socio-economic level?

(a) Low

(b) Medium

(c) High

(2) Regarding law 18.471 on responsible ownership and AW, what is your level of knowledge?

(a) Heard about the law

(b) Have read the text of law and understand its reach

(c) Have read the text of the law and do not understand its reach

(d) Have no knowledge of the law

(3) During your work activities, what's the number of animals with signs of abuse that you observed?

(a) One per week

(b) More than one per week

(c) One per month

(d) Never

(4) If you receive a pet owner with an animal with clear signs of abuse?

(a) You give medical attention to the animal

(b) You give medical attention to animal and advise the owner

(c) You report the owner to the authorities

(d) You deny medical attention

(e) Do not have the proper tools to proceed in this situation

7c Food industry (meat, dairy or other)

(1) In your work area, how do you evaluate the influence of AW on the quality of the final product?

(a) No influence

(b) Moderate influence

(c) Great influence

(2) In your work area, staff training on AW is:

(a) Frequent

(b) Sporadic

(c) Inexistent

(3) Do you consider that the training on AW in your work area was:

(a) Useful and improved animal handling

(b) Useful and improved workers' performances

(c) Useful but soon forgotten

(d) Requested by the client

(e) Offered by third parties

(f) None of the previous

(4) How important is AW and good animal handling practices for the veterinarian and plant staff?

(a) High

(b) Medium

(c) Low 


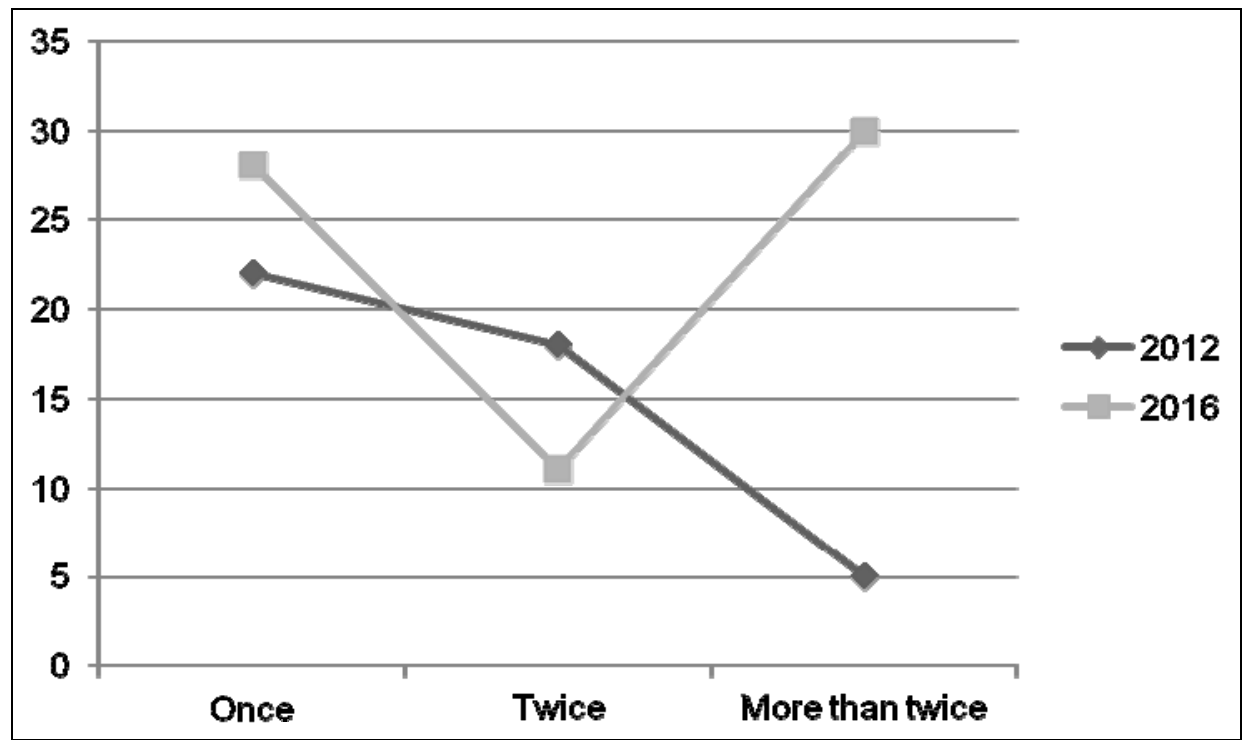

Fig. 1 Comparison of percentage of veterinarians who attended AW trainings or courses by times in 2012 to 2016.

Table 2 Herding tools used to move animals on livestock farms in Uruguay, visited by veterinarians who answered the 2016 questionnaire.

\begin{tabular}{ll}
\hline Herding tool & $\%$ \\
\hline Electric prod & 41.0 \\
Sticks & 52.1 \\
Shouting & 70.4 \\
Flags & 77.5 \\
Dogs & 61.9 \\
Did not answer & 12.1 \\
\hline
\end{tabular}

More than one herding tool can be used in the farm.

high percentage of flag use (77.5\%) stands out, nevertheless there is a similarly high use of electric prods (41\%), sticks (52.1\%) and occurrence of shouting (70.4\%). These results match with the previous responses provided by farm owners prior to good animal handling practices trainings [6]. This could indicate that the need to reduce or avoid the use of these kinds of elements, which can hurt the animal's integrity, was not sufficiently addressed in the education campaigns.

Nevertheless, these farms are advised by a veterinarian, who declared attending at least one AW course and that the information received was useful for their job. It is evident that there is a need to improve the training received by veterinarians, focusing on the great importance of their role as promoter and positive example for farmers. In this case, Fvet-UdelaR must play its role in creating and promoting knowledge in health and AW areas.

About $75.9 \%$ of veterinarians who worked in the food industry consider that AW has a great influence on the quality of the final product [16]. Nevertheless, $47.8 \%$ of the industry workers received training sporadically, $26.8 \%$ frequently and $25.5 \%$ never. According with the veterinarians, these trainings not only improved the way that plant workers treat the animals (42\%), but also improved the overall workers' performance (41.3\%).

\section{Conclusions}

The majority of veterinarians surveyed seemed to be sensible with the topic and had assisted to at least one AW course. At the same time, they considered the knowledge acquired with great utility and demonstrated a will to gain more knowledge on the topic. It is necessary to continue working towards the inclusion of AW in the veterinary curricula, as to continue with the promotion of good handling practices and AW principles, based on scientific research and on the national and international regulations at all levels.

\section{Acknowledgments}

The authors thank Teresita Alonso from the 
Programa de Educación Continua de Facultad de Veterinaria de la Universidad de la República and all the veterinarians who kindly sent in their responses for the questionnaire.

\section{References}

[1] César, D., and Huertas, S. 2007. "The Diffusion and Training Experience in Animal Welfare and Good Management Practices at the Beef Cattle Chain in Uruguay.” In Proceeding of the Regional Seminar on Animal Welfare: Strategies for Dissemination of Good Animal Husbandry, 85-93. (in Spanish)

[2] World Organization for Animal Health (OIE). 2009. "Collaborating Centre on Animal Welfare and Livestock Production Systems.” Accessed August 18, 2016. http://www.oie.int/en/our-scientific-expertise/collaboratin g-centres/list-of-centres/.

[3] World Organization for Animal Health (OIE). 2012. "Recommendations of the OIE on the Expected Minimum Competencies of Newly Licensed Veterinarians to Ensure High Quality National Veterinary Services.” Accessed 2012. http://www.oie.int/fileadmin/Home/esp/Support_to_OIE_ Members/Edu_Vet_AHG/day_1/DAYONE-B-esp-VC.pd f. (in Spanish)

[4] World Organization for Animal Health (OIE). 2013. "Basic Curriculum of Veterinary Training." OIE Guidelines. Accessed September, 2013. http://www.oie.int/Plan_de_Estudios_Basico_de_Formac ion_Veterinaria.pdf. (in Spanish)

[5] Herranz, F. V., Villaplana, F., and Cadavid, A. M. 2016. "Animal Welfare and Animal Protection: A Strategic Vision for the Veterinary Profession.” Accessed November 17, 2016. http://www.colvet.es/node/2099.

[6] Gil, A., and Huertas, S. 2007. "Dissemination of Animal Welfare Principles and Good Management Practices along the Whole Meat Chain.” In Proceeding of the Regional Seminar on Animal Welfare: Strategies for Dissemination of Good Animal Husbandry, 109-16. (in Spanish)

[7] Huertas, S. 2012. "Continuing Training and Education of Graduate Veterinarians in Animal Welfare.” In Proceeding of the Third OIE Global Conference on Animal Welfare.

[8] European Commission. 2012. "EU Strategy on Animal
Welfare.” Accessed November 10, 2016. https://ec.europa.eu/food/animals/welfare/strategy_en.

[9] Gallo, C., Cajiao, M. N., Tadich, T., Calderón, J., and Pereira, V. 2016. "Incorporating Animal Welfare into the Professional Profile of the Veterinarian in Latin America.” In Inclusion of Animal Welfare Topics in the Curricula of Veterinary Medicine Colleges in Latin America, edited by Taylor, J. Panama: Pan American Association of Veterinary Sciences, 75-91. (in Spanish)

[10] World Organization for Animal Health (OIE). 2012. "Regional Animal Welfare Strategy for the Americas." Accessed November, 2012. http://www.rr-americas.oie.int/fileadmin/Documents/ANI MAL_WELFARE/ER_23CR.pdf.

[11] Galindo, F., Huertas, S., and Gallo, C. 2014. "OIE Strategy for the Teaching of Animal Welfare in the Americas.” In Animal Welfare and Veterinary Education in Latin America. Mexico: Guadalajara University, 63-70. (in Spanish)

[12] Ministry of Livestock Agriculture and Fisheries (MGAP). 2010. "National Veterinary Census of Uruguay 2010.” Accessed December 2, 2016. http://archivo.presidencia.gub.uy/sci/noticias/2011/02/Ce nso_Nacional_Veterinario_Uruguay.pdf. (in Spanish)

[13] Huertas, S. M., César, D., Piaggio, J. and Gil, A. 2015. "Evaluation of Education of Veterinarians in Animal Welfare Issues.” Journal of Agricultural Science and Technology A 5 (2): 136-40.

[14] Barcos, L. O. 2014. "Presentation of the Regional Animal Welfare for the Americas." World Organization for Animal Health (OIE). Accessed July, 2016. http://www.bienestaranimal.org.uy/files/01\%20-\%20Pres entacion\%20de\%20la\%20Estrategia\%20Regional\%20BA _Barcos.pdf. (in Spanish)

[15] Ministry of Livestock Agriculture and Fisheries (MGAP). 2016. “Agricultural Statistical Yearbook." Area of Agricultural Statistics (DIEA), MGAP. Accessed September 12, 2016. http://www.mgap.gub.uy/sites/default/files/exportaciones _e_importaciones_pesqueras_por_ano_-_anuario_diea_2 016.pdf. (in Spanish)

[16] Huertas, S. 2007. "Quality Assurance in the Meat Chain.” In Proceeding of the Regional Seminar on Animal Welfare: Strategies for Dissemination of Good Animal Husbandry, 101-9. (in Spanish) 